\title{
C atalizadores de manganeso sintetizados por autocombustión y coprecipitación y su empleo en la oxidación del 2-propanol
}

\author{
María Haidy C astaño, R afael M olina, Sonia M oreno* \\ Estado Sólido y Catálisis Ambiental (ESCA), Departamento de Química, Facultad de Ciencias, \\ Universidad Nacional de Colombia, Bogotá, Colombia
}

\begin{abstract}
Resumen
Se sintetizaron óxidos mixtos de manganeso a través de las metodologías de autocombustión y coprecipitación manteniendo constantes las relaciones $\mathrm{Mn}^{2+} / \mathrm{Mg}^{2+}=1$ y $\mathrm{M}^{2+} / \mathrm{M}^{3+}=3$, las cuales son características de los óxidos obtenidos a través de la descomposición térmica de precursores del tipo de la hidrotalcita. Los catalizadores se caracterizaron mediante las técnicas de fluorescencia de rayos X (FRX), difracción de rayos X (DRX), microscopía electrónica de barrido (SEM), adsorción de $\mathrm{N}_{2}$, reducción a temperatura programada y se evaluaron en la oxidación catalítica del 2-propanol. Los resultados evidenciaron que el empleo de la autocombustión como método de síntesis permite generar óxidos con propiedades estructurales, texturales, de reducción-oxidación (redox) y catalíticas similares a las obtenidas para el óxido preparado a través del precursor tipo hidrotalcita. El desempeño catalítico de los óxidos mixtos se asoció directamente con sus propiedades redox. El óxido de manganeso obtenido por autocombustión se depositó sobre monolitos metálicos de FeCrAlloy y se evaluó el efecto de la adherencia de la fase activa por medio del recubrimiento previo de los materiales con alúmina coloidal. La oxidación del 2-propanol sobre el monolito evidenció que no hay pérdida de actividad cuando el catalizador está sobre un material estructurado.
\end{abstract}

Palabras clave: hidrotalcita, autocombustión, coprecipitación, monolito, COV.

Self-combustion and C 0-precipitation manganese catalysts and their use in the oxidation of 2-propanol

\begin{abstract}
A bstract
Manganese mixed oxides were synthesized by self-combustion and co-precipitation, maintaining constant the relationship $\mathrm{Mn}^{2+} / \mathrm{Mg}^{2+}=1$ and $\mathrm{M}^{2+} / \mathrm{M}^{3+}=3$, which are characteristic of the oxides obtained by thermal decomposition of hydrotalcite-like precursors. The catalysts were characterized using X-ray fluorescence (XRF), X-ray diffraction (XRD), scanning electron microscopy (SEM), $\mathrm{N}_{2}$ adsorption, and temperature-programmed reduction, and they were evaluated in the catalytic oxidation of 2 -propanol. The results showed that the use of such self-combustion synthesis method allows generating oxides with structural, textural, redox and catalytic properties similar to those obtained for the oxide prepared via a hydrotalcite-like precursor. The catalytic performance of mixed oxides was directly related to their redox properties. We evaluated the manganese oxide obtained by self-combustion deposited on Fecralloy metal monoliths, as well as the effect of the adhesion of the active phase of the precoat materials using colloidal alumina. The oxidation of 2-propanol over the monolith showed that there is no loss of activity when a catalyst is on a structured material.
\end{abstract}

Key words: Hydrotalcite, self-combustion, co-precipitation, monolith, VOC.

\section{Introducción}

La oxidación catalítica es una de las tecnologías empleadas para el control de los compuestos orgánicos volátiles (E veraert \& Baeyens, 2004). Entre los sistemas catalíticos utilizados, los óxidos metálicos son de gran interés por ser económicos y por su versatilidad a la hora de aplicarlos en diferentes reacciones de oxidación. Aunque existen diversos métodos de síntesis para la preparación de óxidos mixtos (Schwarz, et al., 1995), el método de coprecipitación de un precursor tipo hidrotalcita es el más empleado.
Los hidróxidos de doble capa (layered double hydroxides, LDH), también conocidos como arcillas aniónicas o compuestos del tipo de la hidrotalcita (HT), representan un grupo de materiales laminares sintéticos cuya fórmula general puede expresarse como

$$
\left[\mathrm{M}^{2+}{ }_{1-\mathrm{x}} \mathrm{M}^{3+}{ }_{\mathrm{x}}(\mathrm{OH})_{2}\right]^{\mathrm{x}+}\left[\mathrm{A}_{\mathrm{x} / \mathrm{n}}^{\mathrm{n}-} \cdot \mathrm{mH}_{2} \mathrm{O}\right]^{\mathrm{x}-},
$$

\section{*Correspondencia:}

Sonia Moreno, smorenog@unal.edu.co

Recibido: 23 de julio de 2014

Aceptado: 19 de enero de 2015 
donde $\mathrm{M}^{2+} \mathrm{y} \mathrm{M}^{3+}$ son cationes divalentes $\mathrm{y}$ trivalentes, respectivamente, $\mathrm{A}^{\mathrm{n}-}$ es un anión y $\mathrm{x}$ debe encontrarse en el intervalo entre 0,2 y 0,33 para preservar la estructura (K ovanda \& J irátová, 2011; X u, et al., 2011). El empleo de precursores tipo hidrotalcita permite generar óxidos mixtos con grandes áreas superficiales (100-300 m²/g), así como interdispersión homogénea de los elementos, dispersión de la fase activa, efectos sinérgicos y carácter básico asociado a la presencia de pares ácido-base $\left(\mathrm{O}^{2-}-\mathrm{M}^{+}\right)$y oxígeno terminales $\left(\mathrm{O}^{2-}\right)$, características útiles en las reacciones de oxidación (Cavani, et al., 1991; Di Cosimo, et al., 1998; Vaccari, 1998).

En la síntesis de hidrotalcitas de manganeso, se ha estudiado la estabilidad térmica del material preparado por coprecipitación empleando $\mathrm{Mg}\left(\mathrm{NO}_{3}\right)_{2}$ y $\mathrm{MnCl}_{2}$ en medio básico. Los estudios por $\mathrm{TPR}-\mathrm{H}_{2}$ han revelado que el $84 \%$ del $\mathrm{Mn}^{2+}$ se oxida a $\mathrm{Mn}^{3+}$ durante la síntesis. Además, el tratamiento térmico a $390{ }^{\circ} \mathrm{C}$ conduce a la formación de un material amorfo, mientras que la calcinación a temperaturas mayores $\left(670\right.$ y $1.000{ }^{\circ} \mathrm{C}$ ) lleva a la cristalización de $\mathrm{MgO}$ y $\mathrm{Mg}_{2} \mathrm{MnO}_{4}$ (Fernández, et al., 1994). En una serie de hidróxidos de doble capa con relaciones $(\mathrm{Mg}+\mathrm{Mn}) / \mathrm{Al}=3$ y $\mathrm{Mg}: \mathrm{Mn}$ de 3:0 a 0:3, sintetizados por el método de coprecipitación, se ha evaluado la incorporación de Mn en las estructuras laminares de Mg-Al. Cuando existe la relación Mg:Mn:Al=2,2:0,8:1, se produce la formación de una única fase hidrotalcita y con el incremento del contenido de $\mathrm{Mn}$ se obtiene la mezcla de las fases $\mathrm{LDH}, \mathrm{Mn}(\mathrm{OH})_{2}$ y $\mathrm{MnCO}_{3}$. Igualmente, los autores han reportado que cuando las hidrotalcitas se calcinan a $500{ }^{\circ} \mathrm{C}$, se genera una solución sólida de MgO y cargas elevadas de $\mathrm{Mn} \mathrm{y}$, además de $\mathrm{MgO}$, se forman las espinelas $\mathrm{Mn}_{3} \mathrm{O}_{4}$ y $\mathrm{MnAl}_{2} \mathrm{O}_{4}$ (Velu, et al., 1999). En la evaluación de la oxidación del tolueno, del etanol y del butanol sobre óxidos mixtos de $\mathrm{Mn}$, Co-Mn y Cu-Mn preparados por la descomposición térmica de hidrotalcitas, los materiales han exhibido un excelente comportamiento en la oxidación de los compuestos orgánicos volátiles (COV), atribuido principalmente a la generación de fases amorfas y ciclos de redox en los óxidos (Aguilera, et al., 2011).

El empleo de hidrotalcitas como precursores de óxidos mixtos es uno de los métodos de síntesis más comunes debido a las propiedades únicas que presentan los materiales finales. Sin embargo, la autocombustión representa otro método de síntesis de gran interés en la preparación de materiales, ya que permite la generación directa de los óxidos mixtos en menores tiempos de síntesis sin la necesidad de formar un precursor para obtener óxidos mixtos con buena distribución y dispersión de las fases activas, así como buenas propiedades texturales. La reacción de autocombustión empleando nitratos metálicos y glicina como combustibles puede describirse a través de la siguiente ecuación general:

$$
\begin{gathered}
M^{v}\left(\mathrm{NO}_{3}\right)_{v}+\left(\frac{5}{9} v \varphi\right) \mathrm{CH}_{2} \mathrm{NH}_{2} \mathrm{CO}_{2} \mathrm{H}+v \frac{5}{4}(\varphi-1) \mathrm{O}_{2} \rightarrow \\
M^{v} \mathrm{O}_{v / 2(S)}+\left(\frac{10}{9} v \varphi\right) \mathrm{CO}_{2(g)}+\frac{25}{18} \varphi H_{2} \mathrm{O}_{(g)}+v\left(\frac{5}{9} \varphi+1\right) / 2 \mathrm{~N}_{2(g)}
\end{gathered}
$$

donde $M$ es un metal de valencia $v$ y $\varphi$ es el oxígeno requerido para la oxidación completa del combustible. Cuando $\varphi=1$ no se requiere oxígeno atmosférico, y $1<\varphi$ $<1$ indican, respectivamente, condiciones ricas y pobres en oxígeno (M ukasyan, et al., 2007).

El medio inicial de reacción (solución acuosa), que permite la mezcla de los reactantes a nivel molecular al igual que las altas temperaturas de reacción que aseguran productos con alta pureza y cristalinidad, la corta duración del proceso y la formación de varios gases que inhiben el crecimiento del tamaño de las partículas y favorecen la síntesis de sólidos con grandes áreas superficiales, son algunos de los factores más importantes que contribuyen a las propiedades únicas de los productos finales sintetizados durante la autocombustión (M ukasyan \& Dinka, 2007; Tahmasebi \& Paydar, 2008).

Por otro lado, el empleo de materiales estructurados como los monolitos ha despertado gran interés en el control de gases contaminantes, entre estos los empleados en los convertidores catalíticos para la transformación de los gases de origen vehicular emitidos al ambiente (Heck, et al., 2001). Entre las ventajas que presentan los monolitos se encuentran la baja pérdida de carga y de presión, una gran superficie por unidad de volumen o masa, un flujo de gases muy uniforme y, en el caso de los monolitos metálicos, una gran conductividad térmica y una notable resistencia mecánica (C ybulski \& Moulijn, 1994).

Los monolitos metálicos están conformados por dos elementos: el soporte, que es el material estructural o sustrato metálico, y el catalizador, que recubre las paredes del monolito y que está compuesto de una fase activa dispersa sobre un soporte. El mayor inconveniente en la preparación de monolitos metálicos es la baja adherencia de la fase activa al sustrato metálico, razón por la cual se utilizan materiales como el FeCrAlloy, que contienen $\mathrm{Al}$, el cual genera una capa de alúmina que permite la adhesión del recubrimiento catalítico después de ser sometido a un tratamiento térmico (I vanova, et al., 2013).

En este trabajo se estudió la síntesis de óxidos de manganeso mediante dos metodologías de síntesis, la coprecipitación y la autocombustión, y se compararon las propiedades estructurales, texturales, redox y catalíticas de los materiales a partir de las técnicas de caracterización empleadas. Igualmente, se prepararon monolitos metálicos cuya fase activa sería el catalizador más activo, lo que, hasta donde se sabe, constituye un método novedoso que no ha sido reportado hasta el momento en la literatura científica.

\section{Procedimiento experimental}

\section{Síntesis de catalizadores}

Coprecipitación. Se sintetizó el óxido mixto Mg-Al-Mn por medio de la descomposición térmica de precursores tipo hidrotalcita obtenido por el método de coprecipitación 
simultánea, en el cual se emplearon las soluciones de los nitratos respectivos conservando las relaciones $\mathrm{M}^{2+} / \mathrm{M}^{3+}=3 \mathrm{y}$ $\mathrm{Mn}^{2+} / \mathrm{Mg}^{2+}=1$.

Para la síntesis se mezclaron los nitratos de $\mathrm{Mg}^{2+}, \mathrm{Al}^{3+} \mathrm{y} \mathrm{Mn}^{2+}$ y se añadieron gota a gota a una solución de $0,2 \mathrm{M}$ de $\mathrm{K}_{2} \mathrm{CO}_{3}$ con agitación constante, manteniendo el pH entre 9,5 y 10,5 (se agregó una solución de $1 \mathrm{M}$ de $\mathrm{NaOH}$ ). El precipitado obtenido se dejó envejecer durante 18 h sin agitación. Posteriormente, el sólido se lavó y se secó a $60^{\circ} \mathrm{C}$ en aire durante 18 h (obtención de la hidrotalcita HTMn) y se calcinó a $500{ }^{\circ} \mathrm{C}$ durante $16 \mathrm{~h}$ para obtener el óxido mixto denominado CPMn (la abreviatura CP indica coprecipitación).

Autocombustión. Se emplearon las soluciones de los nitratos de $\mathrm{Mg}\left(\mathrm{NO}_{3}\right)_{2} \cdot 6 \mathrm{H}_{2} \mathrm{O}, \mathrm{Al}\left(\mathrm{NO}_{3}\right)_{3} \cdot 9 \mathrm{H}_{2} \mathrm{O}$ y $\mathrm{Mn}\left(\mathrm{NO}_{3}\right)_{2} \cdot 6 \mathrm{H}_{2} \mathrm{O}$, que actuaron como oxidantes, y una solución de glicina $\left[\mathrm{CH}_{2} \mathrm{NH}_{2} \mathrm{COOH}\right]$, la cual se empleó como combustible. Se mantuvieron constantes la relaciones molares $\mathrm{M}^{2+} / \mathrm{M}^{3+}=3$, $\mathrm{Mn}^{2+} / \mathrm{Mg}^{2+}=1$, y una relación combustible/oxidante (glicina/ nitratos) igual a 1,0 .

El óxido mixto Mg-Al-Mn se obtuvo por la adición de los nitratos respectivos a la glicina con agitación constante. La solución resultante se sometió a evaporación lenta hasta la obtención de un gel que se calentó a alrededor de $100{ }^{\circ} \mathrm{C}$, lo que dio el impulso necesario para llevar a cabo el proceso de ignición. Una vez se alcanzó la reacción de ignición, se obtuvo un polvo que se calcinó a $500{ }^{\circ} \mathrm{C}$ durante $4 \mathrm{~h}$ para eliminar los residuos carbonáceos y obtener finalmente el óxido ACMn (abreviatura en la que AC significa autocombustión).

\section{Catalizadores estructurados}

Se prepararon monolitos de $3 \mathrm{~cm}$ de longitud y 1,6 cm de diámetro empleando láminas de FeCrAlloy (Fe: 72,6 \%; Cr: 22 \%; Al: 4,8 \%; Si: 0,3 \%; y Y: 0,3 \%). Se enrollaron láminas lisas y corrugadas de FeCrAlloy en torno a un eje para obtener el material estructurado. Por último, los monolitos generados se calcinaron a $900{ }^{\circ} \mathrm{C}$ durante $22 \mathrm{~h}$ para inducir la segregación de whiskers de $\alpha-\mathrm{Al}_{2} \mathrm{O}_{3}$ en la superficie que aseguraran la adherencia de la fase activa (A guero, et al., 2011; Pérez, et al., 2010).

Para depositar la fase activa sobre el monolito, se empleó el método de deposición catalítica por vía húmeda, washcoating, y se evaluó el efecto del recubrimiento directo del soporte estructurado con la suspensión de la fase activa y el recubrimiento previo con un iniciador antes de emplear la suspensión de la fase activa (Barbero, et al., 2008). Antes de realizar el recubrimiento, el óxido ACMn se molió en un molino de bolas a $400 \mathrm{rpm}$ durante $5 \mathrm{~h}$ para obtener un tamaño de partícula de $27 \mu \mathrm{m}$. La distribución del tamaño de partícula se determinó en un analizador láser Mastersizer 2000 de Malvern Instruments.

Se preparó una suspensión al 12 \% del catalizador ACMn molido con 2,1 \% de alcohol de polivinilo, 8,4 \% de Nyacol (20\% de alúmina coloidal, la cual actúa como iniciador) y $77,5 \%$ de agua para realizar los recubrimientos.
Los monolitos se sumergieron luego en la suspensión durante $1 \mathrm{~min}$ y se retiraron a una velocidad de $3 \mathrm{~cm}$ por $\mathrm{min}^{-1}$; el exceso de suspensión se eliminó por centrifugación a 400 rpm durante $3 \mathrm{~min}$; los monolitos se secaron a $120^{\circ} \mathrm{C}$ durante $1 \mathrm{~h}$ después de cada inmersión. Por último, los monolitos recubiertos se calcinaron a $500{ }^{\circ} \mathrm{C}$ durante $2 \mathrm{~h}$ para obtener el catalizador M-Mn.

Con el fin de mejorar la adherencia de la suspensión en el monolito, se hizo un recubrimiento con Nyacol antes de emplear la suspensión de ACMn. Para esto, el monolito se sumergió durante 1 min en la suspensión de Nyacol, se secó a $120^{\circ} \mathrm{C}$ durante $1 \mathrm{~h}$ y se calcinó a $500^{\circ} \mathrm{C}$ durante $2 \mathrm{~h}$. Posteriormente, se hizo el recubrimiento con la suspensión de ACMn siguiendo el procedimiento descrito anteriormente para así obtener el material M-Ny-Mn.

Con el fin de conocer el pH adecuado para ajustar la suspensión, se midió el potencial Vs. el pH en el óxido mixto ACMn. Las medidas del punto isoeléctrico se llevaron a cabo en un Zeta Meter System 3.0, empleando $20 \mathrm{mg}$ de ACMn disperso en $250 \mathrm{ml}$ de una solución de 0,001 M de $\mathrm{KCl}$, y el pH se ajustó empleando soluciones de 0,01 M de $\mathrm{KOH}$ y $\mathrm{HCl}$.

El punto isoeléctrico del catalizador ACMn fue 10,2. El valor más alto del potencial fue de $36 \mathrm{mV}$ para un $\mathrm{pH}$ de 5 , por lo que las suspensiones se prepararon ajustando un $\mathrm{pH}$ de 5 con $\mathrm{HNO}_{3}$ diluido, el cual es un valor alejado del punto isoeléctrico del catalizador. De esta manera se incrementó la repulsión entre las partículas previniendo su aglomeración y obteniendo suspensiones más estables (A guero, et al., 2011).

La prueba de adherencia se llevó a cabo con el fin de conocer la pérdida de peso de los monolitos después de ser sometidos a un baño de ultrasonido. Para esto, los monolitos recubiertos se sumergieron en $25 \mathrm{ml}$ de éter de petróleo y se llevaron a un baño de ultrasonido durante $30 \mathrm{~min}$. Posteriormente, los monolitos se secaron a $120^{\circ} \mathrm{C}$ durante $1 \mathrm{~h}$ y se calcinaron a $500{ }^{\circ} \mathrm{C}$ durante $2 \mathrm{~h}$. Las pérdidas de peso de los recubrimientos se calcularon por la diferencia de peso de los monolitos antes y después del tratamiento con ultrasonido (A guero, et al., 2011).

\section{Caracterización}

El análisis químico de los materiales en polvo se hizo en un espectrómetro de fluorescencia de rayos X MagixPro PW2440 Phillips con una potencia máxima de 4 KW.

La composición de las fases presentes en los óxidos se determinó mediante la técnica de difracción de rayos $\mathrm{X}$ (DRX) en un equipo SHIMADZU LAB- X XRD- 6000 provisto de un ánodo de cobre $(\lambda=1,54060 \AA)$, empleando una velocidad de $1 \circ \theta \min ^{-1}$ y un tamaño de paso de $0,02{ }^{\circ} \Theta$. El tamaño de partícula se calculó con la ecuación de Scherrer, usando la señal más intensa de cada óxido. 
La morfología de los óxidos de manganeso en polvo se estudió por microscopía electrónica de barrido empleando un aparato FEI QUANTA 200 en modo de alto vacío y con un voltaje de $30 \mathrm{kV}$. Para los materiales estructurados se empleó un equipo HITACHI S-2700 con voltaje de $15 \mathrm{kV}$

Para la determinación del área superficial de los óxidos, se obtuvieron isotermas de adsorción-desorción de $\mathrm{N}_{2}$ a $77 \mathrm{~K}$, empleando un equipo Micromeritics ASAP 2020.

Los análisis de reducción a temperatura programada con hidrógeno (TPR-H2) se hicieron en un equipo Chembet 3000 Quantachrome equipado con un detector de conductividad térmica. Los materiales (100 mg) se sometieron previamente a desgasificación a $400{ }^{\circ} \mathrm{C}$ durante $1 \mathrm{~h}$ en presencia de Ar y se redujeron empleando una mezcla de $\mathrm{H}_{2} / \mathrm{Ar}$ al $10 \%$ (v/v), un flujo de $30 \mathrm{ml} / \mathrm{min}^{-1} \mathrm{y}$ una rampa de calentamiento de $10^{\circ} \mathrm{C} / \mathrm{min}$. Los consumos de hidrógeno se calcularon a partir de una curva de calibración de CuO.

\section{Actividad catalítica}

Los catalizadores en polvo y el catalizador estructurado se evaluaron en la oxidación catalítica del 2-propanol. Se empleó un reactor de lecho fijo en $\mathrm{U}$, que opera en flujo continuo y a presión atmosférica, con un caudal total de $280 \mathrm{ml} / \mathrm{min}, 0,200$ g de catalizador (tamizado $<250 \mu \mathrm{m}$ ) y una concentración de 1.000 ppm de 2-propanol.

Los catalizadores se activaron con un flujo de $280 \mathrm{ml} / \mathrm{min}$ de una mezcla de $21 \%$ de $\mathrm{O}_{2}$ en $\mathrm{He}$ a $400{ }^{\circ} \mathrm{C}$ durante $1 \mathrm{~h}$ y se estabilizaron durante $1 \mathrm{~h}$ con la corriente de la mezcla de oxígeno y el 2-propanol a $400{ }^{\circ} \mathrm{C}$. La curva de ignición se obtuvo por enfriamiento a $1,5{ }^{\circ} \mathrm{C} / \mathrm{min}$ desde $400{ }^{\circ} \mathrm{C}$ hasta $50{ }^{\circ} \mathrm{C}$. Los reactivos y productos de la reacción de oxidación se analizaron en línea por espectrometría de masas (espectrómetro de masas Balzers Omnistar), siguiendo las señales m/z 45,43,41,18 y 44 asignadas respectivamente al 2-propanol y a los principales productos de reacción: acetona, propeno, agua y dióxido de carbono.

\section{R esultados y discusión}

\section{Caracterización del precursor hidrotalcita}

El patrón de difracción de la hidrotalcita de manganeso, HTMn, se presenta en la figura 1, donde se compara con el patrón de difracción típico de una hidrotalcita de $\mathrm{Mg}$-Al. La presencia de las señales intensas en 11,3, 22,6 y 34,4 ${ }^{\circ} 2 \theta$ indican la formación de una estructura laminar bien cristalizada con simetría 3R (Tsyganok \& Sayari, 2006). Los parámetros cristalográficos $a$ y $c$ de las dos estructuras laminares HTMn y HTMgAl, se calcularon a partir de las reflexiones (110) y (003) de los perfiles, donde $a=2 d_{(110)}$ y $c=3 d_{(003)}$, respectivamente (Evans \& Slade, 2006; Pérez, et al., 2011). El parámetro $a$, que corresponde a la distancia entre dos cationes, presentó el mismo valor para HTMn y para $\mathrm{HTMgAl}(a=0.307 \mathrm{~nm})$, lo que sugiere que la presencia de manganeso no perturba dicha distancia. Sin embargo, el parámetro $c$, que corresponde al espesor

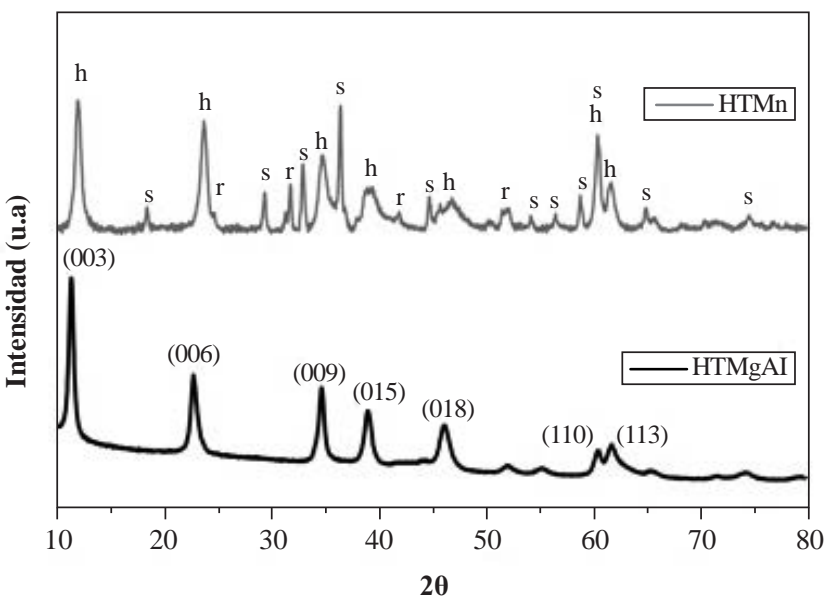

Figura 1. Difracción de rayos X (DRX) de las hidrotalcitas de Mn y de $\mathrm{Mg}-\mathrm{Al}(\mathrm{h})$ fase hidrotalcita, (s) $\mathrm{Mn}_{3} \mathrm{O}_{4}$, (r) $\mathrm{MnCO}_{3}$

de tres láminas del tipo de la brucita más su espacio interlaminar, reveló un valor ligeramente menor en HTMn $(2,26 \mathrm{~nm})$ que en HTMgAl $(2,34 \mathrm{~nm})$. Este resultado se atribuyó a un incremento de las fuerzas electrostáticas entre las láminas y las interláminas, efecto que sólo es posible si se encuentran los iones $\mathrm{Mn}^{3+}$ presentes en las láminas brucíticas (Velu, et al., 1999). La presencia de estos iones dentro de la estructura laminar explicaría, igualmente, por qué el Mn no afecta el parámetro $a$ de la hidrotalcita HTMn.

La hidrotalcita de Mn reveló las mismas señales de la hidrotalcita de referencia $(\mathrm{MgAl})$ y otras señales atribuidas a la formación de las fases hausmanita $\mathrm{Mn}_{3} \mathrm{O}_{4}$ (JCPDS 24-0734) y rodocrosita $\mathrm{MnCO}_{3}$ (JCPDS 44-1472). La formación de $\mathrm{Mn}_{3} \mathrm{O}_{4}$ probablemente se debe a que parte del $\mathrm{Mn}^{2+}$ se oxida hasta $\mathrm{Mn}^{3+}$ en medio básico (condiciones de síntesis empleadas). Estos resultados concuerdan con los valores de los parámetros cristalográficos calculados para esta hidrotalcita (Fernández, et al., 1994; Velu, et al., 1999).

La micrografía de HTMn obtenida por SEM se muestra en la figura 2a. Es evidente la formación de una estructura laminar, lo que confirma la presencia de una estructura del tipo hidrotalcita.

\section{Caracterización de los óxidos mixtos}

Los patrones de difracción del óxido mixto obtenido a través de la descomposición térmica de la hidrotalcita de manganeso, CPMn, y el obtenido por autocombustión, ACMn, presentaron señales propias de espinelas de manganeso $\mathrm{Mn}_{3}$ $\mathrm{O}_{4}, \mathrm{Mn}_{2} \mathrm{MgO}_{4}, \mathrm{Mn}_{2} \mathrm{AlO}_{4}$ y $\mathrm{MgMn}_{1.75} \mathrm{Al}_{0.25} \mathrm{O}_{4}$ (JCPDS No 240734, JCPDS No 23-0392, JCPDS No 29-0881 y JCPDS No 01-075-0528, respectivamente), cuyos máximos coincidieron, sin ser posible su asignación exacta (figura 3 ). Se observó, además, la formación de la periclasa $\mathrm{MgO}$, fase normalmente obtenida tras la descomposición térmica de los precursores tipo hidrotalcita. 


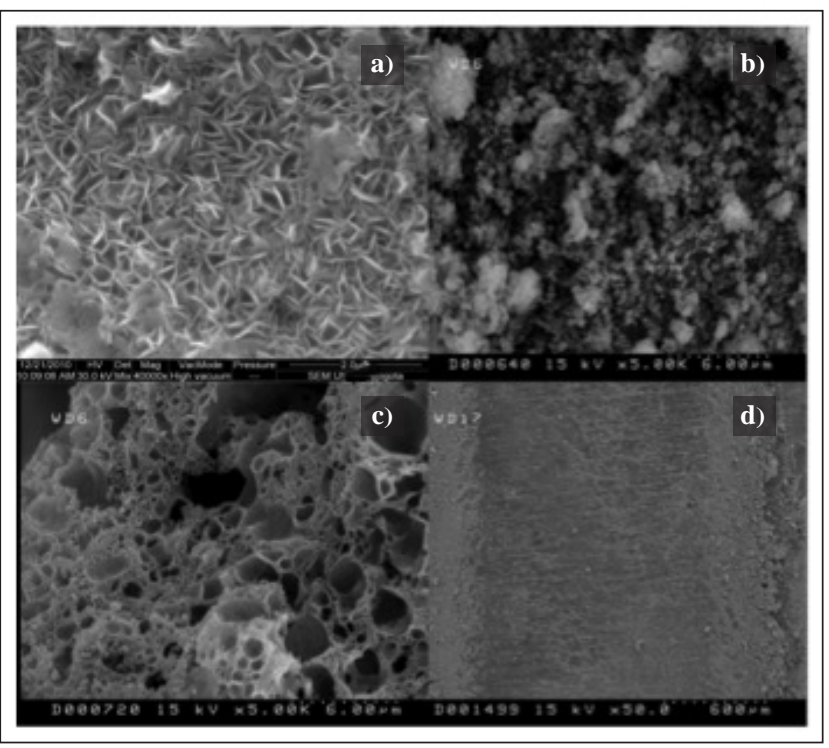

Figura 2. Micrografías de microscopía electrónica de barrido (SEM) de los materiales. a) HTMn, b) CPMn, c) ACMn, d) M-NyMn

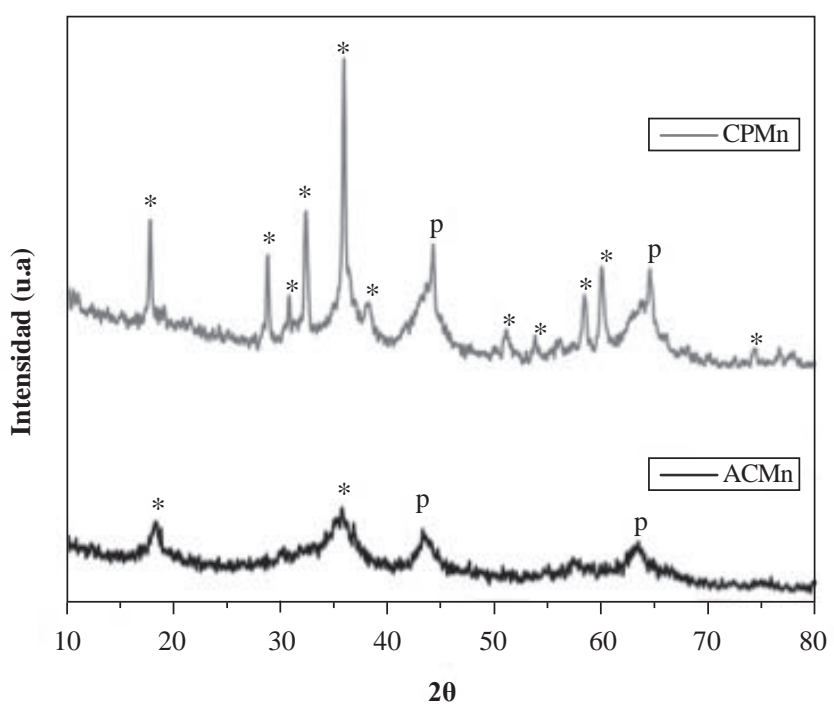

Figura 3. Difracción de rayos $\mathrm{X}$ (DRX) de los óxidos mixtos de manganeso. (p) $\mathrm{MgO}(*) \mathrm{Mn}_{3} \mathrm{O}_{4}, \mathrm{Mn}_{2} \mathrm{MgO}_{4}, \mathrm{Mn}_{2} \mathrm{AlO}_{4}$, $\mathrm{MgMn}_{1.75} \mathrm{Al}_{0.25} \mathrm{O}_{4}$

Los tamaños de partícula de los óxidos, estimados mediante la ecuación de Scherrer y la señal más intensa (211), se presentan en la tabla 1. El óxido mixto obtenido por autocombustión presentó un tamaño de partícula mucho menor comparado con el obtenido en el óxido sintetizado a través del precursor tipo hidrotalcita, lo cual evidencia que la autocombustión alcanza materiales con tamaños de partícula muy pequeños en razón de la gran evolución de productos gaseosos que se produce durante la síntesis, lo que evita el contacto entre partículas y mejora la dispersión y distribución de la fase activa.
En las estructuras del tipo de la hidrotalcita la conservación de las relaciones $\mathrm{M}^{2+} / \mathrm{M}^{3+}=3$ y $\mathrm{M}^{2+} / \mathrm{Mg}^{2+}=1$ permitió obtener catalizadores con un excelente desempeño catalítico en la oxidación de los COV, como se ha demostrado en la oxidación catalítica del tolueno (Aguilera, et al., 2011; C astaño, et al., 2013; Pérez, et al., 2011).

El análisis químico por FRX de los sólidos se presenta igualmente en la tabla 1. En los dos óxidos mixtos obtenidos se observa que las relaciones $\mathrm{M}^{2+} / \mathrm{M}^{3+}$ y $\mathrm{Mn}^{2+} / \mathrm{Mg}^{2+}$ correspondieron a las relaciones nominales establecidas, por lo que fue posible realizar una incorporación satisfactoria de las especies a través de las metodologías de coprecipitación y de autocombustión.

Las curvas de adsorción-desorción de $\mathrm{N}_{2}$ sobre los dos óxidos de CPMn y ACMn revelaron el carácter mesoporoso de los materiales. El área BET fue la misma en los dos catalizadores (tabla 1), sin que se viera afectada por el método de síntesis empleado. En el óxido CPMn el área se atribuye a la formación de poros resultantes de la destrucción de los espacios laminares de la hidrotalcita después de la calcinación (pérdida de agua y carbonatos) (A guilera, et al., 2011). En el caso del óxido ACMn, la generación de gases como el $\mathrm{CO}_{2}$ y el $\mathrm{H}_{2} \mathrm{O}$ durante la combustión resulta en un efecto plantilla que conduce a la obtención de materiales con gran porosidad.

Las figuras $2 \mathrm{~b}$ y $2 \mathrm{c}$ presentan las micrografías SEM del CPMn y del ACMn y allí se evidencia que el óxido mixto proveniente de la autocombustión presentó una estructura altamente porosa, con poros en el rango de los micrómetros, como resultado de la gran cantidad de productos gaseosos generados durante la síntesis del material. Aunque el ACMn evidencia la presencia de macroporos, la contribución de los mesoporos es evidente de acuerdo a los resultados de la isoterma de adsorción-desorción, con lo que se obtuvo un área BET similar a la del CPMn, el cual es un material fundamentalmente mesoporoso.

Los perfiles de TPR- $\mathrm{H}_{2}$ de los óxidos de manganeso aparecen en la figura 4. En general, son perfiles bastantes complejos en razón de los múltiples estados de oxidación del manganeso y a los diferentes ambientes químicos en los que se puede encontrar. Sin embargo, el perfil de reducción puede clasificarse en tres zonas principales, una de baja temperatura $\left(<400{ }^{\circ} \mathrm{C}\right)$, otra de temperatura intermedia $\left(400{ }^{\circ} \mathrm{C}-500^{\circ} \mathrm{C}\right)$ y una tercera de alta temperatura $\left(>500^{\circ} \mathrm{C}\right)$.

En la primera zona se verifican los procesos reductivos de especies de $\mathrm{MnOx}$ con diversos tamaños de partícula, y la transición de $\mathrm{Mn}_{2} \mathrm{O}_{3}$ a $\mathrm{Mn}_{3} \mathrm{O}_{4}$. La segunda zona se atribuye al paso de la especie $\mathrm{Mn}_{3} \mathrm{O}_{4}$ hacia la forma reducida del manganeso $\mathrm{MnO}$ (Aguilera, et al., 2011; C raciun, et al., 2003; Döbber, et al., 2004; Stobbe, et al., 1999). En la tercera zona las señales se atribuyen a la presencia de fases tipo 
Tabla 1. Composición química, tamaño de partícula, área específica $\left(\mathrm{S}_{\mathrm{BET}}\right)$, consumos de hidrógeno y estado promedio de oxidación (AOS) de los óxidos

\begin{tabular}{|c|c|c|c|c|c|c|c|}
\hline \multirow{3}{*}{ Sólido } & \multicolumn{2}{|c|}{ A nálisis químico } & \multirow{3}{*}{$\begin{array}{c}\text { Tamaño de } \\
\text { partícula }( \pm 3 n m)\end{array}$} & \multirow{3}{*}{$\begin{array}{c}S_{B E T} \\
\left( \pm 10 \mathrm{~m}^{2} g^{-1}\right)\end{array}$} & \multicolumn{3}{|c|}{ Propiedades redox } \\
\hline & \multirow{2}{*}{$M^{2+} / M^{3+}$} & \multirow{2}{*}{$\mathrm{Mn}^{2+} / \mathrm{Mg}^{2+}$} & & & \multicolumn{2}{|c|}{ Consumo $\mathrm{H}_{2}(\mathrm{mmol} / \mathrm{g}) \pm 0,1$} & \multirow{2}{*}{ AOS } \\
\hline & & & & & Total & $<400^{\circ} \mathrm{C}$ & \\
\hline CPMn & 2,9 & 1,2 & 34 & 186 & 3,7 & 1,4 & 3,2 \\
\hline ACMn & 2,9 & 1,3 & 6 & 184 & 4,1 & 1,6 & 3,1 \\
\hline
\end{tabular}

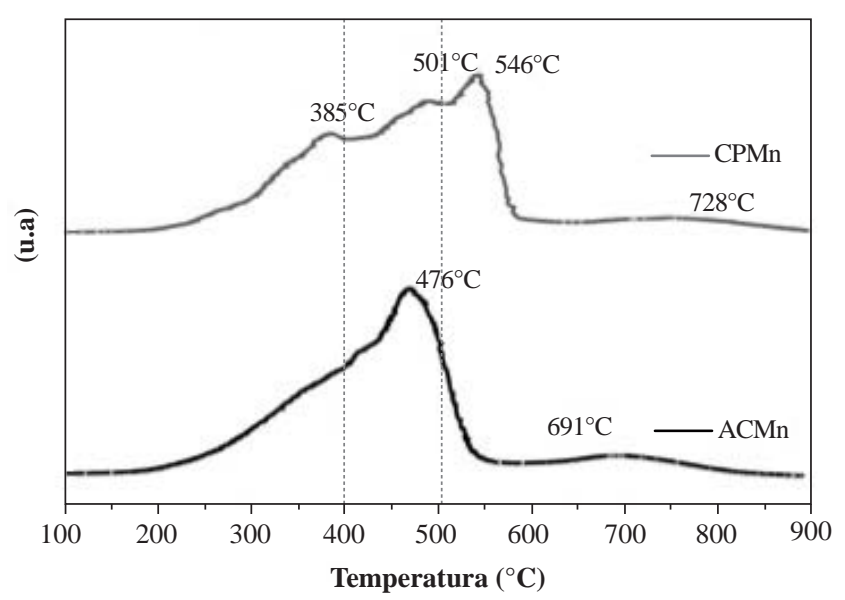

Figura 4. Perfiles de TPR- $\mathrm{H}_{2}$ de los óxidos mixtos de manganeso

espinela, como se evidenció por DRX, y a la reducción del manganeso que hace parte de la solución sólida (A guilera, et al., 2011).

El óxido mixto ACMn presentó una señal más intensa en la reducción de $\mathrm{Mn}_{3} \mathrm{O}_{4} \rightarrow \mathrm{MnO}$, lo que puede asociarse a un mejor desempeño catalítico en reacciones de oxidación, ya que, como se ha establecido, los óxidos de manganeso con átomos de $\mathrm{Mn}$ en diferentes estados de oxidación, como es el caso de la hausmanita $\mathrm{Mn}_{3} \mathrm{O}_{4}$, propician un ambiente de movilidad electrónica óptimo para efectuar una reacción de óxido-reducción sobre su superficie (Santos, et al., 2009).

Los consumos de hidrógeno totales se presentan en la tabla 1 , en la cual se reporta igualmente el consumo de hidrógeno a temperaturas menores de $400{ }^{\circ} \mathrm{C}$ debido a que es allí donde se verifica el comportamiento redox de los sólidos en condiciones de reacción catalítica. Al comparar los resultados de los óxidos de manganeso generados por las dos metodologías se observó que el óxido CPMn presentó, en promedio, las temperaturas más altas de reducción y el menor consumo de hidrógeno total, lo que sugiere la presencia de especies de manganeso de menor acceso o menor capacidad de reducción debido, probablemente, a mayores tamaños de partícula, como se evidenció por DRX. Sin embargo, el consumo de hidrógeno a temperaturas menores de $400{ }^{\circ} \mathrm{C}$ fue similar en los dos óxidos, lo que indicaría que es posible obtener óxidos con propiedades redox similares bajo las condiciones de reacción con ambas metodologías.
El estado promedio de oxidación (average oxidation state, AOS) de los óxidos de manganeso presentes en los catalizadores se indica también en la tabla 1. Dichos valores se estimaron mediante la siguiente reacción:

$$
\mathrm{MnO}_{\mathrm{x}}+(x-1) \mathrm{H}_{2} \rightarrow \mathrm{MnO}+(x-1) \mathrm{H}_{2} \mathrm{O},
$$

donde se asumió que el $\mathrm{MnO}_{\mathrm{x}}$ presente se reduce hasta $\mathrm{MnO}$, y el estado de oxidación promedio $(2 x)$ puede calcularse a partir del área bajo la curva de todos los picos en el análisis por TPR- $\mathrm{H}_{2}$ y del porcentaje de manganeso presente en el catalizador (Pérez, H. et al., 2011; Pérez, H., et al., (2013); Torres, et al., 2011).

Los resultados relativos al AOS sugieren que tanto en el CPMn como en el ACMn los iones de Mn se encontraban en una mezcla de estados de oxidación de $3+$ y $2+$, por lo que se constató que es posible obtener óxidos de manganeso con el mismo estado de oxidación mediante las dos metodologías.

La presencia de Mn en diferentes estados de oxidación es favorable, ya que se reconoce ampliamente que los óxidos que presentan diferentes estados de oxidación son más activos en las reacciones de oxidación total (Baldi, et al., 1998; K im \& Shim, 2010).

Teniendo en cuenta los resultados de las diferentes caracterizaciones, es claro que mediante el método de autocombustión fue posible obtener óxidos mixtos con propiedades semejantes a los generados con el empleo de precursores del tipo de la hidrotalcita, pero en mucho menor tiempo de síntesis ( $25 \mathrm{~h}$ Vs. 48 h), por lo que el óxido ACMn se seleccionó como fase activa en la obtención de catalizadores estructurados.

\section{Caracterización del material estructurado}

Considerando el comportamiento catalítico del óxido de manganeso obtenido por el método de autocombustión, resulta interesante evaluar la potencialidad de soportar dicho catalizador sobre un monolito metálico y estudiar el desempeño catalítico de este sistema.

En el desarrollo de sistemas catalíticos estructurados es de vital importancia la adherencia de la suspensión sobre el sustrato metálico, donde la retención del recubrimiento sobre el monolito puede llevarse a cabo a través de dos mecanismos: por un anclaje entre las mismas partículas del recubrimiento, o por medio de las irregularidades o rugosidad de la superficie del soporte, la cual se produce por el tratamiento térmico 
del monolito de FeCrAlloy, el cual permite la formación de una superficie rugosa de whiskers de óxido de aluminio que facilita el anclaje del recubrimiento sobre la superficie del monolito (A guero, et al., 2011; Avila, et al., 2005).

En la figura 5 se presenta la evolución de la masa de la suspensión retenida sobre el monolito en función de los recubrimientos realizados. El número de recubrimientos es función de la masa de fase activa que debe soportarse sobre los monolitos. Se obtuvieron alrededor de 0,180 g de ACMn después del proceso de calcinación sobre los materiales estructurados con un $60 \%$ de adherencia, tal y como se indica en la tabla 2. La masa obtenida fue muy cercana a la empleada cuando se tiene el catalizador de manganeso en polvo para la oxidación catalítica del 2-propanol.

Como se ha planteado, la adherencia del catalizador sobre el soporte es muy importante y, aunque la superficie del sustrato metálico se somete a tratamiento térmico, algunas veces la afinidad química entre el monolito y la fase activa no es suficiente y estos tienden a separarse, lo que resulta en una baja adherencia. Esta baja adherencia puede contrarrestarse mediante un recubrimiento del monolito con una capa intermedia de alúmina, denominada comúnmente iniciador, sobre la cual se deposita posteriormente la fase activa. En general, la función del iniciador es actuar como "pegamento" entre el sustrato metálico y el catalizador aumentando la adherencia entre ellos (M ontebelli, et al., 2014; Visconti, 2012), y generar sitios de enlace entre los whiskers de alúmina y el catalizador.

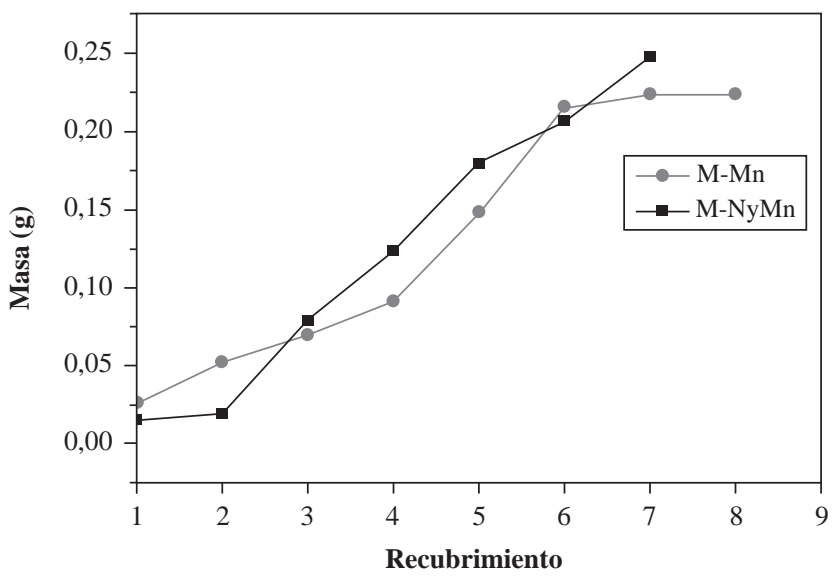

Figura 5. Inmersiones realizadas Vs. masa de suspensión retenida sobre los monolitos

Tabla 2. Masa de catalizador retenida después de calcinar a $500^{\circ} \mathrm{C}$ y prueba de adherencia

\begin{tabular}{|c|c|c|c|}
\hline Catalizador & $\begin{array}{c}\text { Masa de } \\
\text { catalizador (g) }\end{array}$ & $\begin{array}{c}\text { Masa de Nyacol (g) } \\
\text { (Ny) }\end{array}$ & $\%$ adherencia \\
\hline M-Mn & 0,186 & ----- & 60 \\
\hline M-NyMn & 0,187 & 0,032 & 78 \\
\hline
\end{tabular}

En la tabla 2 se evidencia el efecto benéfico del empleo de un iniciador en el sistema catalítico usado, comprobado mediante pruebas de adherencia, pues este aumentó la adherencia de la fase activa ( $\mathrm{MnMgAlO}$ ) sobre el monolito.

Aunque el porcentaje de adherencia incrementó, en la figura 5 se aprecia que el empleo de Nyacol como iniciador disminuyó ligeramente la masa de suspensión retenida durante el primer y el segundo recubrimiento. Sin embargo, también se observa que esta tendencia cambió a partir del tercer recubrimiento en el que la masa aumentó con el número de inmersiones.

El comportamiento durante el primer y el segundo recubrimiento se atribuye a una pérdida de rugosidad de la superficie del FeCrAlloy calcinado cuando se emplea un iniciador, debido a que se retiene una capa de alúmina proveniente del Nyacol que mejora la adherencia, pero hace más difícil la deposición de una mayor cantidad del óxido ACMn si se tiene en cuenta que uno de los mecanismos de retención de la fase activa sucede por efecto de la rugosidad de la superficie del monolito, tal como ha sido reportado por B arbero, et al. (2008), quienes señalan que una mayor adherencia con el empleo de un iniciador no compensa la baja cantidad de sólido retenido.

A partir del tercer recubrimiento, la superficie del material estructurado queda constituida principalmente por capas del catalizador de $\mathrm{Mn}$ (ACMn), desapareciendo así el efecto del iniciador, lo que explicaría el aumento de masa de la fase activa, en la cual el mecanismo de anclaje entre las partículas del recubrimiento prevalece y, por lo tanto, permite una mayor carga, como ocurre con el sistema M-Mn, en el cual se verifica que a mayor número de inmersiones mayor retención del catalizador.

La figura 6 presenta la apariencia de los materiales estructurados finales después de realizar los recubrimientos con la suspensión de ACMn y su posterior calcinación a $500{ }^{\circ} \mathrm{C}$ durante dos horas.

La figura $2 \mathrm{~d}$ muestra la micrografía SEM de M-NyMn después de los recubrimientos y el proceso de calcinación; se aprecia un recubrimiento exitoso del monolito con el catalizador ACMn, evidenciando las posibilidades que se abren al emplear este tipo de catalizadores como fases activas en materiales estructurados.

\section{Actividad catalítica}

Es reconocido que la oxidación de 2-propanol se inicia con la adsorción en fase gaseosa del alcohol como especie 2-propóxido y que, posteriormente, ocurre la descomposición de esta especie a través de dos reacciones paralelas: la deshidrogenación hasta acetona, en la cual se requieren sitios básicos fuertes, y la deshidratación hasta propeno, en la cual están involucrados sitios ácidos fuertes y sitios básicos débiles (Liu \& Yang, 2008; Manríquez, et al., 


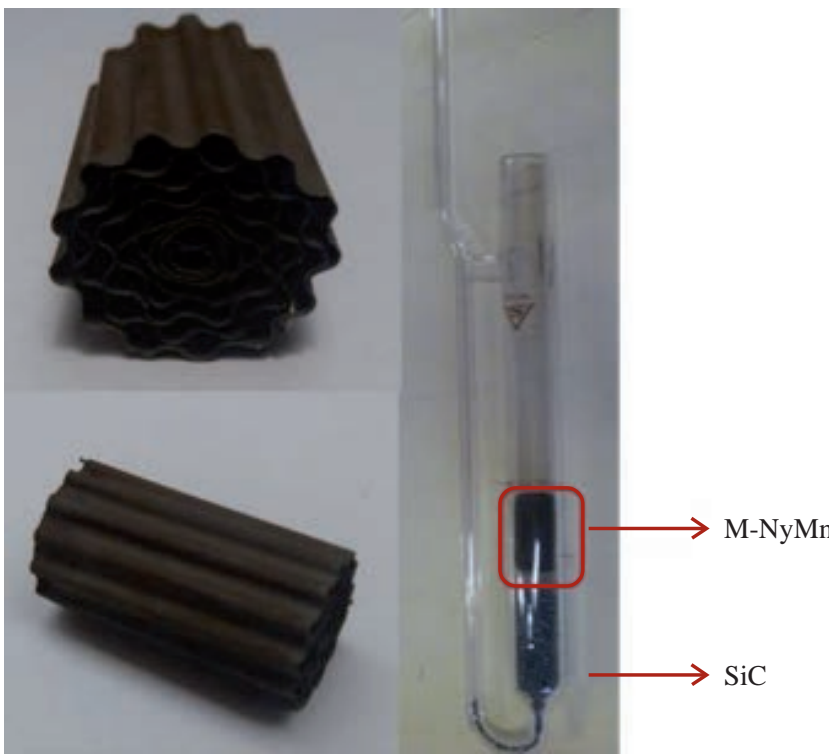

Figura 6. Apariencia del monolito M-NyMn de FeCrAlloy calcinado y montaje en el reactor de lecho fijo

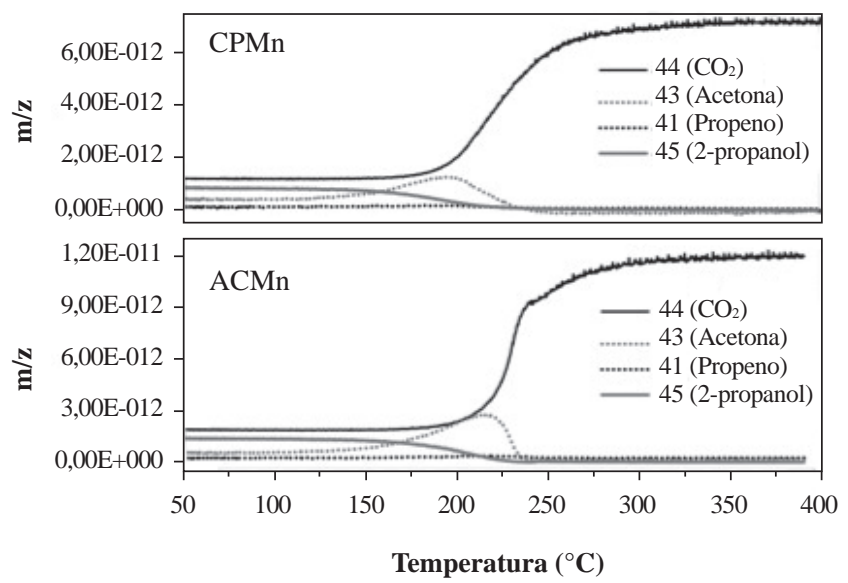

Figura 7. Distribución de especies durante la oxidación del 2propanol

2004). La figura 7 presenta la evolución de las diferentes especies obtenidas en la oxidación del 2-propanol sobre ACMn y CPMn. La distribución de los productos en los dos catalizadores fue similar. Se evidencia la formación de acetona como intermediario de la reacción de acuerdo con las propiedades básicas y redox de los dos óxidos, sin que se obtuviera propeno al incrementar la temperatura.

Se obtuvieron las curvas light-off a partir de los resultados de $\mathrm{m} / \mathrm{z}$ versus temperatura, transformando la señal $\mathrm{m} / \mathrm{z}$ en términos de conversión y empleando los valores de $\mathrm{T}_{50} \mathrm{y} \mathrm{T}_{90}$ (temperatura necesaria para alcanzar el $50 \%$ y el $90 \%$ de conversión del 2-propanol, respectivamente) como medida de actividad. Los resultados se presentan en la tabla 3 y en la figura 8. El comportamiento catalítico de los óxidos de manganeso obtenidos mediante las dos metodologías de síntesis fue muy semejante, lo cual concordó con los resultados de TPR-H $\mathrm{H}_{2}$. Sin embargo, se registró una ligera diferencia en el $100 \%$ de la conversión del 2-propanol hasta $\mathrm{CO}_{2}$, pues sobre el CPMn este valor se alcanzó a $320^{\circ} \mathrm{C}$ mientras que sobre ACMn se logró a $300^{\circ} \mathrm{C}$. Se sabe que la oxidación de los COV puede llevarse a cabo a través de un mecanismo redox de Mars Van Krevelan, en el cual la molécula orgánica es oxidada por los oxígenos de red más cercanos a la superficie del óxido, causando, a la vez, la reducción de este último, el cual vuelve a oxidarse por la presencia de oxígeno en fase gaseosa (Hosseini, et al., 2012), lo que evidencia la existencia de una relación entre el comportamiento catalítico y las propiedades de redox de los catalizadores.

Por otra parte, el desempeño catalítico del material estructurado reveló una distribución de productos muy semejante a la obtenida sobre ACMn en polvo, lo que demuestra la formación de acetona como producto intermediario y la formación de $\mathrm{CO}_{2}$ y agua como productos finales de la oxidación total del 2-propanol. Al igual que el sólido ACMn, el $100 \%$ de la conversión del 2-propanol sobre M-NyMn se alcanzó a una temperatura de $300{ }^{\circ} \mathrm{C}$ y las temperaturas requeridas para alcanzar el 50 \% y el 90 \% de conversión del COV (tabla

Tabla 3. Desempeño catalítico de los catalizadores en polvo y del catalizador estructurado

\begin{tabular}{lccccc}
\hline \multirow{2}{*}{ Sólido } & \multicolumn{5}{c}{ Oxidación del 2-propanol } \\
& \multicolumn{2}{c}{ Conversión } & \multicolumn{2}{c}{ Conversión a C $\mathbf{~}_{\mathbf{2}}$} & A cetona \\
\cline { 2 - 6 } & $\mathbf{T}_{\mathbf{5 0}}\left({ }^{\circ} \mathbf{C}\right)$ & $\mathbf{T}_{90}\left({ }^{\circ} \mathbf{C}\right)$ & $\mathbf{T}_{\mathbf{5 0}}\left({ }^{\circ} \mathrm{C}\right)$ & $\mathbf{T}_{\mathbf{9 0}}\left({ }^{\circ} \mathbf{C}\right)$ & $\mathbf{T}_{\mathbf{M}}\left({ }^{\circ} \mathrm{C}\right)$ \\
\hline CPMn & 190 & 230 & 224 & 270 & 196 \\
\hline ACMn & 186 & 220 & 220 & 254 & 196 \\
\hline M-NyMn & 190 & 220 & 220 & 270 & 200 \\
\hline
\end{tabular}

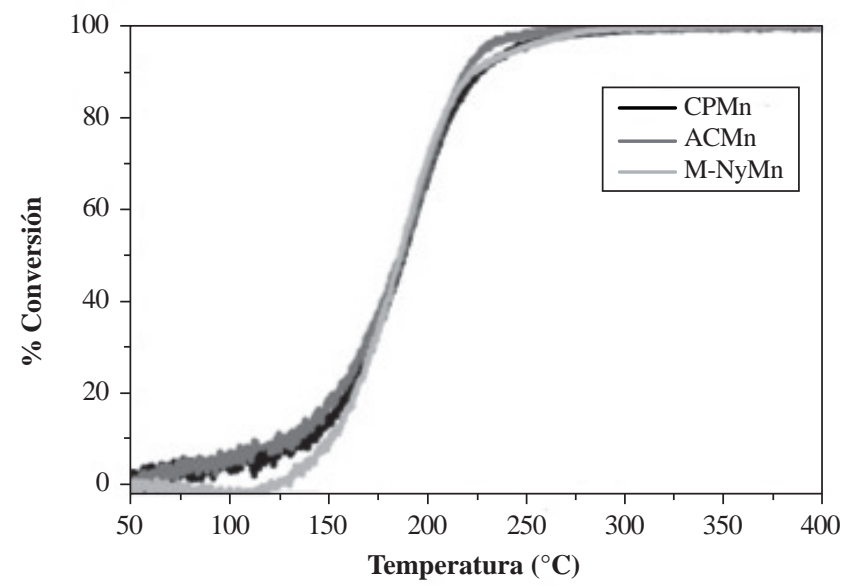

Figura 8. Conversión del 2-propanol sobre los catalizadores en polvo y el catalizador estructurado 
3) fueron las mismas que para el catalizador en polvo. Estos resultados demuestran que la actividad catalítica del ACMn en la oxidación del 2-propanol se conservó en el monolito y el desempeño catalítico no disminuyó por soportarlo sobre un sistema estructurado, como sí se ha reportado para otros sistemas en la literatura científica (C iambelli, et al., 1999; Sanabria, et al., 2010).

\section{Conclusiones}

El empleo de la autocombustión como método de síntesis permitió la generación de óxidos mixtos en menores tiempos de síntesis ( $(5 \mathrm{~h})$ y con propiedades texturales, redox y catalíticas semejantes o superiores a las obtenidas por la metodología tradicional de coprecipitación ( $~ 48 \mathrm{~h})$.

Los óxidos mixtos de manganeso preparados mediante las dos metodologías de síntesis presentaron un desempeño catalítico semejante en la oxidación del 2-propanol, lo que se atribuyó a propiedades redox equivalentes.

El empleo de alúmina coloidal sobre FeCrAlloy mejoró la adherencia del óxido de manganeso en un 30 \% con respecto al material en el que no se usó Nyacol.

El óxido de manganeso ACMn sobre el monolito de FeCrAlloy presentó un desempeño catalítico comparable al que tiene en polvo, lo que permite confirmar su potencial para ser empleado como fase activa en materiales estructurados para reacciones de oxidación de diferentes COVs.

\section{Agradecimientos}

Los autores agradecen al profesor Mario Montes de la Universidad del País Vasco (España) por el apoyo durante la realización de la pasantía doctoral en su grupo de investigación.

\section{Conflicto de intereses}

Los autores declaran no tener ningún conflicto de intereses.

\section{Bibliografía}

Aguero, F. N., Barbero, B. P.,Almeida, L . C ., M ontes, M ., C adús, L. E. (2011). MnOx supported on metallic monoliths for the combustion of volatile organic compounds. Chemical Engineering Journal. 166 (1): 218-223.

Aguilera, D. A., Pérez, A., M olina, R., Moreno, S. (2011). Cu$\mathrm{Mn}$ and $\mathrm{Co}-\mathrm{Mn}$ catalysts synthesized from hydrotalcites and their use in the oxidation of VOCs. Applied Catalysis, B. 104 (1-2): 144-150.

Ávila, P., M ontes, M ., M iró, E . E . (2005). Monolithic reactors for environmental applications: A review on preparation technologies. Chemical Engineering Journal. 109 (1-3): 11-36.

Baldi, M ., Finocchio, E ., M ilella, F., Busca, G . (1998). Catalytic combustion of $\mathrm{C} 3$ hydrocarbons and oxygenates over Mn3O4. Applied Catalysis B: Environmental. 16 (1): 43-51.

Barbero, B. P., C osta-Almeida, L., Sanz, O., M orales, M. R ., Cadus, L . E ., M ontes, M . (2008). Washcoating of metallic monoliths with a $\mathrm{MnCu}$ catalyst for catalytic combustion of volatile organic compounds. Chemical Engineering Journal. 139 (2): 430-435.
C astaño, M. H., M olina, R ., M oreno, S. (2013). Mn-Co-Al-Mg mixed oxides by auto-combustion method and their use as catalysts in the total oxidation of toluene. Journal of Molecular Catalysis A: Chemical. 370 (0): 167-174.

Cavani, F., Trifirò, F., Vaccari, A. (1991). Hydrotalcite-type anionic clays: Preparation, properties and applications. Catalysis Today. 11 (2): 173-301.

Ciambelli, P., Palma, V., Tikhov, S. F., Sadykov, V. A., I supova, L. A., Lisi, L. (1999). Catalytic activity of powder and monolith perovskites in methane combustion. Catalysis Today. 47 (1-4): 199-207.

Craciun, R., Nentwick, B., Hadjiivanov, K., Knözinger, H. (2003). Structure and redox properties of $\mathrm{MnOx} / \mathrm{Yttrium}-$ stabilized zirconia (YSZ) catalyst and its use in CO and CH4 oxidation. Appl. Catal., A. 243 (1): 67-79.

C ybulski, A . \& M oulijn, J . A . (1994). Monoliths in Heterogeneous Catalysis. Catalysis Reviews. 36 (2): 179-270.

Di Cosimo, J. I., Díez, V. K., Xu, M., Iglesia, E., Apesteguía, C. R . (1998). Structure and Surface and Catalytic Properties of Mg-Al Basic Oxides. Journal of Catalysis. 178 (2): 499-510.

Döbber, D., K ießling, D., Schmitz, W., Wendt, G . (2004). MnOx/ $\mathrm{ZrO} 2$ catalysts for the total oxidation of methane and chloromethane. Appl. Catal., B. 52 (2): 135-143.

E vans, D. \& Slade, R. T. (2006). Structural Aspects of Layered Double Hydroxides. In X. Duan \& D. Evans (Eds.), Layered Double Hydroxides (Vol. 119, pp. 1-87): Springer Berlin Heidelberg.

E veraert, K. \& Baeyens, J. (2004). Catalytic combustion of volatile organic compounds. Journal of Hazardous Materials. 109 (1-3): 113-139.

Fernández, J. M., Barriga, C., Ulibarri, M.-A., L abajos, F. M., Rives, V. (1994). Preparation and thermal stability of manganese-containing hydrotalcite, $\left[\mathrm{Mg}_{0.75} \mathrm{MnII}_{0.04} \mathrm{MnIII}_{0.21}\right.$ $\left.(\mathrm{OH})_{2}\right]\left(\mathrm{CO}_{3}\right)_{0.11} \mathrm{nH}_{2} \mathrm{O}$. Journal of Materials Chemistry. 4 (7): 1117-1121.

Heck, R. M ., G ulati, S., Farrauto, R. J . (2001). The application of monoliths for gas phase catalytic reactions. Chemical Engineering Journal. 82 (1-3): 149-156.

Hosseini, S. A., Niaei, A., Salari, D., Nabavi, S. R. (2012). Nanocrystalline AMn2O4 ( $\mathrm{A}=\mathrm{Co}, \mathrm{Ni}, \mathrm{Cu})$ spinels for remediation of volatile organic compounds - synthesis, characterization and catalytic performance. Ceramics International. 38 (2): 1655-1661.

I vanova, S., Pérez, A., Centeno, M . Á., O driozola, J . A . (2013). Chapter 9 - Structured Catalysts for Volatile Organic Compound Removal. In S. L. Suib (Ed.), New and Future Developments in Catalysis. Amsterdam: Elsevier. pp. 233-256.

Kim, S. C. \& Shim, W. G. (2010). Catalytic combustion of VOCs over a series of manganese oxide catalysts. Applied Catalysis B: Environmental. 98 (3-4): 180-185.

K ovanda, F. \& J irátová, K. (2011). Supported layered double hydroxide-related mixed oxides and their application in the total oxidation of volatile organic compounds. Applied Clay Science. 53 (2): 305-316. 
L iu, S. Y. \& Yang, S. M . (2008). Complete oxidation of 2-propanol over gold-based catalysts supported on metal oxides. Applied Catalysis A: General. 334 (1-2): 92-99.

Manríquez, M. E ., L ópez, T., G ómez, R ., Navarrete, J . (2004). Preparation of $\mathrm{TiO} 2-\mathrm{ZrO} 2$ mixed oxides with controlled acid-basic properties. Journal of Molecular Catalysis A: Chemical. 220 (2): 229-237.

M ontebelli, A., Visconti, C. G., Groppi, G., Tronconi, E., Cristiani, C., Ferreira, C., K ohler, S. (2014). Methods for the catalytic activation of metallic structured substrates. Catalysis Science \& Technology. 4 (9): 2846-2870.

M ukasyan, A \& D Dinka, P. (2007). Novel approaches to solutioncombustion synthesis of nanomaterials. International Journal of Self-Propagating High-Temperature Synthesis. 16 (1): 23-35.

Mukasyan, A. S., Epstein, P., Dinka, P. (2007). Solution combustion synthesis of nanomaterials. Proceedings of the Combustion Institute. 31 (2): 1789-1795.

Pérez, A., L amonier, J .-F., Giraudon, J .-M., Molina, R., Moreno, S. (2011). Catalytic activity of $\mathrm{Co}-\mathrm{Mg}$ mixed oxides in the VOC oxidation: Effects of ultrasonic assisted in the synthesis. Catalysis Today. 176 (1): 286-291.

Pérez, H., Navarro, P., Delgado, J . J ., M ontes, M. (2011). MnSBA15 catalysts prepared by impregnation: Influence of the manganese precursor. Applied Catalysis A: General. 400 (1-2): 238-248.

Pérez, H ., Navarro, P., M ontes, M . (2010). Deposition of SBA-15 layers on Fecralloy monoliths by washcoating. Chemical Engineering Journal. 158 (2): 325-332.

Pérez, H., Navarro, P., Torres, G., Sanz, O., Montes, M. (2013) Evaluation of manganese OMS-like cryptomelane supported on SBA-15 in the oxidation of ethyl acetate. Catalysis Today. 212: 149-156.

Sanabria, N. R ., Á vila, P., Yates, M ., R asmussen, S. B ., M olina, R., M oreno, S. (2010). Mechanical and textural properties of extruded materials manufactured with $\mathrm{AlFe}$ and $\mathrm{AlCeFe}$ pillared bentonites. Applied Clay Science. 47 (3-4): 283-289.

Santos, V., Pereira, M „, Ó rfão, J „, Figueiredo, J . (2009). Synthesis and Characterization of Manganese Oxide Catalysts for the Total Oxidation of Ethyl Acetate. Top. Catal. 52 (5): 470-481.

Schwarz, J . A ., Contescu, C ., Contescu, A . (1995). Methods for Preparation of Catalytic Materials. Chemical Reviews. 95 (3): 477-510.

Stobbe, E. R ., de Boer, B. A., G eus, J . W. (1999). The reduction and oxidation behaviour of manganese oxides. Catal. Today. 47 (1-4): 161-167.

Tahmasebi, K. \& Paydar, M. H. (2008). The effect of starch addition on solution combustion synthesis of Al2O3$\mathrm{ZrO} 2$ nanocomposite powder using urea as fuel. Materials Chemistry and Physics. 109 (1): 156-163.

Torres, J. Q., Giraudon, J .-M., L amonier, J .-F. (2011). Formaldehyde total oxidation over mesoporous $\mathrm{MnOx}$ catalysts. Catalysis Today. 176 (1): 277-280.

Tsyganok, A \& Sayari,A. (2006). Incorporation of transition metals into $\mathrm{Mg}-\mathrm{Al}$ layered double hydroxides: Coprecipitation of cations vs. their pre-complexation with an anionic chelator. Journal of Solid State Chemistry. 179 (6): 1830-1841.

Vaccari, A. (1998). Preparation and catalytic properties of cationic and anionic clays. Catalysis Today. 41 (1-3): 53-71.

Velu, S., Shah, N., J yothi, T. M., Sivasanker, S. (1999). Effect of manganese substitution on the physicochemical properties and catalytic toluene oxidation activities of $\mathrm{Mg}-\mathrm{Al}$ layered double hydroxides. Microporous and Mesoporous Materials. 33 (1-3): 61-75.

Visconti, C . G . (2012). Alumina: A Key-Component of Structured Catalysts for Process Intensification. Transactions of the Indian Ceramic Society. 71 (3): 123-136.

Xu, Z. P., Zhang, J., Adebajo, M. O., Zhang, H., Zhou, C. (2011). Catalytic applications of layered double hydroxides and derivatives. Applied Clay Science. 53 (2): 139-150. 